\title{
Apuntes sobre la tecnología educativa, a propósito de los 50 años de la Facultad de Ciencias de la Educación de la Universidad Tecnológica de Pereira*
}

Volumen $5 \mathrm{~N} .044$ enero - junio de 2018

ISSN: 0122-4328

ISSN-E: 2619-6069

pp. 89-102
Notes on Educational

Technology, on the

Occasion of the 50th

Anniversary of the

School of Education

Sciences of Universidad

Tecnológica de Pereira

\author{
Notas sobre tecnologia \\ educacional por ocasião \\ do $50^{\circ}$ aniversário \\ da Faculdade de \\ Ciências da Educação \\ da Universidade \\ Tecnológica de Pereira
}

John Jaime Correa Ramírez"

Natalia Agudelo Castañeda"*

Christian Javier Niño Posada

Fecha de recepción: 17-06-2016

Fecha de aprobación: 14-07-2018

\section{PARA CITAR ESTE ARTÍCULO}

Correa, J., Agudelo, N. y Niño, C. (2018). Apuntes sobre la tecnología educativa, a propósito de los 50 años de la Facultad de Ciencias de la Educación de la Universidad Tecnológica de Pereira. Nodos y Nudos, 5(44), 89-102.

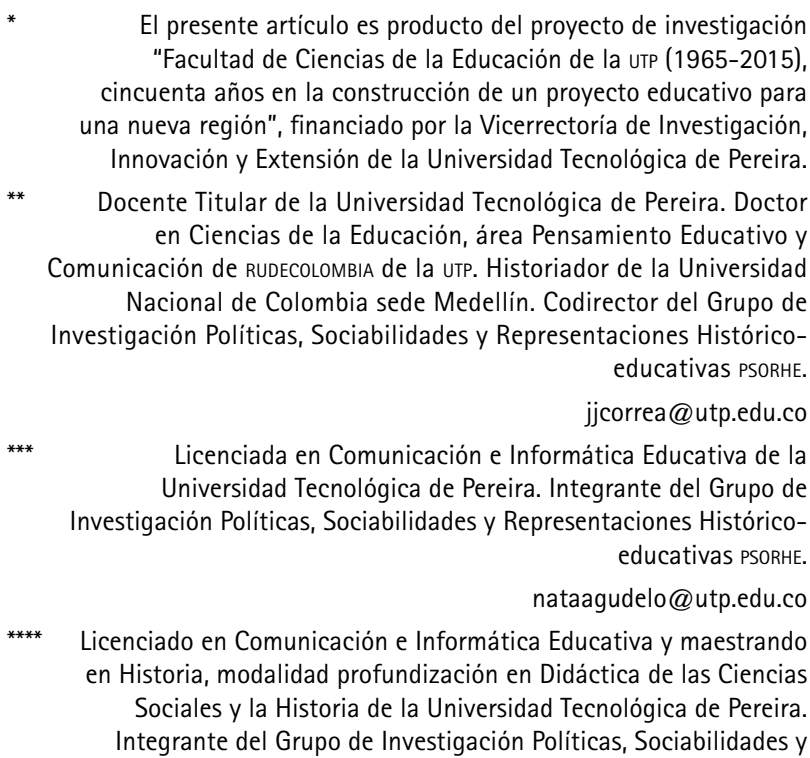
Representaciones Histórico-educativas PSORHE. 


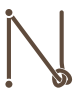

Volumen $5 \mathrm{~N} .{ }^{\circ} 44$ enero - junio de 2018 ISSN: 0122-4328 ISSN-E: 2619-6069 pp. 89-102

\section{RESUMEN}

El objetivo del presente artículo es aportar a la comprensión sobre la consolidación del campo de la tecnología educativa en el interior de la Facultad de Ciencias de la Educación (FCE) de la Universidad Tecnológica de Pereira (UTP), desde una perspectiva histórica, por medio de una interpretación crítica de fuentes primarias en las que se encuentran: los programas de estudio de las licenciaturas a abordar, la legislación que los regula, entrevistas a egresados y profesores jubilados de estos. Este artículo es producto de la investigación "Facultad de Ciencias de la Educación UTP (1967-2017): 50 años en la construcción de un proyecto educativo para una nueva región", en medio de la cual se pudo evidenciar cómo esta línea fue constitutiva en la formación de licenciados en la FCE y que ha prevalecido pese a las reformas educativas de esta. La metodología implementada fue el enfoque cualitativo y el método histórico-crítico, compuesto por dos grandes fases: la realización de un balance historiográfico y crítica de fuentes a partir de entrevistas o grupos focales a quienes fueran estudiantes y profesores de cada programa.

Palabras clave: tecnologia educativa; modernización curricular; educación

\section{ABSTRACT}

The purpose of this paper is to contribute to the understanding of the consolidation of the field of Educational Technology at the School of Education Sciences of Universidad Tecnológica de Pereira from a historical perspective, through a critical interpretation of primary sources, including: the curricula of the degrees to be addressed, the legislation that regulates them, and interviews with graduates and retired teachers of those programs. This paper is the result of a research titled: "UTP School of education Sciences (19672017): 50 years in the construction of an educational project for a new region", during which it was shown how this line contributed to the training of teachers at the School of Education Sciences and how it has prevailed despite the educational reforms thereto. The methodology implemented to develop the research was the qualitative approach and the historical-critical method, composed of two major phases: the first one is a historiographic balance, and the second one is a review of sources based on interviews or focus groups with former students and professors of each program.

Keywords: educational technology; curriculum modernization; education

\section{RESUMO}

0 objetivo deste artigo é contribuir para a compreensão da consolidação do campo da Tecnologia Educacional dentro da Faculdade de Ciências da Educação da Universidade Tecnológica de Pereira, a partir de uma perspectiva histórica, através de uma interpretação crítica de fontes primárias, as quais são: os programas de estudo dos graus a serem abordados, a legislação que os regula, entrevistas com alunos formados e seus professores já aposentados. Este artigo é o resultado da investigação "Faculdade de Educação UTP (1967-2017): 50 anos 
na construção de um projeto educacional para uma nova região". Evidenciamos como esta linha de pesquisa foi constitutiva na formação de licenciados na FCE e que prevaleceu apesar de suas reformas educacionais. A metodologia utilizada para o desenvolvimento da pesquisa foi a abordagem qualitativa e o método histórico-crítico, que compreende duas fases: em primeiro lugar, realizando um balanço historiográfico e em segundo, uma crítica de fonte a partir da realização de entrevistas ou grupos focais com estudantes e professores de cada programa.

Palavras-chave: tecnologia educacional; modernização curricular; educação

\section{Introducción: un breve contexto internacional y nacional}

En la década de 1960, Colombia empezó a preocuparse por ejecutar proyectos de modernización de su estructura institucional, política y económica, como una apuesta por seguirle el paso al desarrollo de los países industrializados. Sin embargo, esto no sucedía por un simple voluntarismo progresista y nacionalista de los gobiernos de turno ni por las exigencias de los movimientos profesorales y estudiantiles, sino que estaba en total consonancia con las directrices que los organismos multilaterales -como la Agencia para el Desarrollo Internacional (USAID), el Banco Mundial (BM), el Banco Interamericano de Desarrollo (BID), el Banco Internacional de Reconstrucción y Fomento (BIRF), y el Banco Internacional de Reconstrucción y Desarrollo (BIRD), por mencionar algunos de los más influyentes-, orientaban para fortalecer los sectores de la industria y la economía de los países en vías de desarrollo.

Bajo este panorama, la educación superior en Colombia empezó a atravesar por una época de reformas que pretendian, por un lado, modernizar su estructura académica y administrativa, y por otro, anclarla al desarrollo económico e industrial del país. Así, en el marco de la Alianza para el Progreso (1961-1971) y su implementación en Colombia durante el Frente Nacional (1958-1974), se publicaron dos documentos claves en la comprensión del ideal de la "universidad para el desarrollo": el informe de Rudolph Atcon, titulado La universidad latinoamericana: clave para un enfoque conjunto del desarrollo coordinado, social y educativo en América Latina, presentado en Tegucigalpa, Honduras, el 31 de marzo de 1961; y la propuesta de reforma de la Universidad Nacional de Colombia hecha por José Félix Patiño Restrepo en su periodo como rector de esta institución entre 1964 y 1966, denominada Hacia la universidad del desarrollo: bases de una politica de reforma universitaria.

Sin embargo, ninguno de estos dos proyectos de reforma lograría transformar el conjunto de la educación superior -con lo que tampoco se transformaría el sistema educativo en general-, puesto que los modelos de enseñanza seguirian siendo los mismos. Un ejemplo de ello lo constituiría el hecho de que se siguiera estudiando la historia de Colombia con el clásico manual de historia de Jesús María Henao y Gerardo Arrubla, titulado Historia de Colombia para la Enseñanza Secundaria, publicado en 1920, pero vigente por casi todo el siglo $\mathrm{xx}$, lo mismo que los trillados catecismos del padre Astete y el Manual de urbanidad de Carreño.

Años más tarde, Philip Coombs, un americano experto en educación, declaró en 1971, después de haber realizado un diagnóstico para el BM, que la educación estaba atravesando por una gran crisis que albergaba unas dificultades de corte estructural, como: analfabetismo, métodos de enseñanza obsoletos, desarticulación con la vida productiva, falta de planeación, entre otros.

Ante ello y para dar salida a estos problemas, Alfonso López Michelsen presentó en su periodo en la Presidencia, entre 1974 y 1978, su plan de desarrollo titulado "Para cerrar la brecha", cuyo objetivo fue "lograr un crecimiento de la economía que haga posible la creación masiva de empleo productivo y, por lo tanto, beneficie de manera especial al cincuenta por ciento más pobre de la sociedad colombiana" (DNP, 1975, p. 3). 
Entre las soluciones que se plantearon para resolver la crisis educativa en Colombia, estaba la preocupación por "la modernización del sistema educacional" (Martínez, Noguera y Castro, 2003, pp. 133-134). Al mismo tiempo, organismos internacionales ya se encontraban impulsando transformaciones especificas que, como en el caso de la Misión Alemana de 1968, repercutió en el devenir de los procesos formativos. En este diagnóstico se planteaba la necesidad del perfeccionamiento educativo de los docentes, el ingreso de más personas al sistema educativo y la renovación curricular (Martínez Boom, 2016a, p. 41). Ante este imperativo, la inserción del componente tecnológico se presentó como el paso que guiaría el avance hacia un nuevo sistema educativo para Colombia.

A partir de 1975 se dio comienzo a la implantación definitiva de la TE con la formulación del Programa de Mejoramiento Cualitativo de la Educación, que fue la base para la posterior reorganización del Ministerio de Educación Nacional en 1976, con la publicación del Decreto 088. Posteriormente, en 1978, la presentación del Proyecto Multinacional de TE (PMTE), gestado por la Organización de Estados Americanos (ofa), que tuvo como objetivo el desarrollo de la capacidad de los Estados miembros de esta organización, para generar y utilizar procesos, métodos e instrumentos tecnológicos en la identificación de sus problemas educativos. Asunto que tuvo gran impacto en Colombia, en especial, en el impulso de procesos educativos mediante el uso de nuevas tecnologías, en especial de la Televisión Educativa (Pérez, s.f., p. 155). En el marco de este proyecto se vendía la idea de que su ejecución ayudaría a que los Estados miembros superaran los rezagos en la carrera por el desarrollo.

Se trata de una especie de cortina de humo, esto es, una crisis de nunca acabar, pues hoy es observable el afán por la obtención de nuevas tecnologías y en sí de la consolidación de un paradigma en el que todos estamos inmersos. Cabe aún pensar si este proceso, a pesar de las propuestas de estos organismos internacionales, ha obedecido también a las reflexiones de quienes se han preocupado en nuestro país por el tema educativo, o si, por el contrario, ha sido una necesidad exógena.
Ahora bien, la Facultad de Ciencias de la Educación (FCE) de la UTP inició con el programa de Licenciatura en Matemáticas y Física, que justificó su origen en una institución con una marcada orientación tecnológica e industrial, bajo la premisa de la enseñanza de las cosas útiles (Acevedo, Gutiérrez y Rodríguez, 2009, p. 209). Como señalan Martínez Boom y Correa (2016), la FCE había sido creada con el imperativo de profesionalizar a los maestros normalistas, tras la creación del departamento de Risaralda, a comienzos del 1967 (UTP, 1965).

En efecto, desde 1969 se hace evidente la urgencia de ampliar la oferta académica de la FCE con nuevos programas para la profesionalización docente. En lo que sigue, conviene señalar que la aplicación de las politicas -que se han mencionado hasta ahora- no ha sido homogénea en todas las instituciones y que recae en la autonomía universitaria asumirlas de manera particular, de acuerdo con sus necesidades. Hecha esta salvedad, la TE tuvo resonancia entre las directivas y profesores de la UTP y por iniciativa de Rodrigo Naranjo, el presbitero Aristóbulo Arias y Ricaurte Murillo, y se propuso la apertura una Licenciatura en Psicopedagogía y ayudas educativas en 1971, por lo que el Consejo Directivo de la UTP decidió enviar una comisión a la Universidad Pedagógica y Tecnológica de Tunja, donde ya habían adelantos en la Psicopedagogía ${ }^{1}$, con la intención de que los docentes delegados se asesoraran en esta área disciplinar a la que se le daría apertura en la UTP.

Luego de que se realizaran ajustes en el pénsum propuesto inicialmente, mediante la Resolución No. 011 de 1971 del Ministerio de Educación, se adoptó el plan de estudios a nivel de Licenciatura en el área de Técnicas Audiovisuales y Psicopedagogía, que para 1975 tendría 223 estudiantes ${ }^{2}$, de un total de 759 estudiantes que sumaban los cinco programas académicos que para ese entonces se ofertaban en la Facultad ${ }^{3}$.

1 Consejo Directivo Universidad Tecnológica de Pereira, Acta No 47. Diciembre 1 de 1971.

2 Esta información se extrajo de los Boletines Estadisticos Históricos de la Universidad Tecnológica de Pereira, que pueden ser consultados en la página web institucional, en la siguiente dirección: http://www.utp.edu.co/estadisticas-e-indicadores/ boletines-estadisticos-historicos

3 En el año de 1975, la Facultad de Ciencias de la Educación tenía los programas de Licenciatura en Matemáticas y Física, Licenciatura en Física y Matemáticas -estos 
De esta manera, empezó a gestarse, dentro de la FCE, una línea académica centrada en el uso de las tecnologías, ligada a la reflexión sobre los modelos pedagógicos y de aprendizaje, estableciéndose de este modo un campo interdisciplinario del conocimiento. El cual se configura como uno de los componentes de la tríada que compone el campo de la tecnología educativa como se verá más adelante.

\section{Surgimiento y desarrollo de un campo disciplinar: 1971-1998}

En el desarrollo de la educación en Colombia y, en especial, de la formación docente, fueron determinantes las misiones extranjeras como la Misión Currie (1950), la Misión Pedagógica Alemana de 1968 -la cual se preocupó por desarrollar materiales (Guías para los maestros), con el fin de facilitar el proceso de enseñanza/aprendizaje-. Bajo la presidencia de Carlos Lleras Restrepo se dictaron una serie de medidas que respondian a las políticas internacionales en materia educativa, las cuales se materializaron en Decretos del Ministerio de Educación Nacional ${ }^{4}$ y en la formulación de nuevos programas académicos. Finalmente, y para el caso que nos ocupa en el presente artículo, el Proyecto Multinacional de te (1978), planteado por la OEA.

Ahora bien, cuando se habla de TE, Clifton Chadwick (2003), un especialista de la OEA en el tema, plantea:

La tecnología educativa está definida como la aplicación de un enfoque organizado y científico con la información concomitante al mejoramiento de la educación en sus variadas manifestaciones y niveles diversos. En su interior existen ciertas áreas: a) la psicología de la enseñanza aprendizaje; b) las técnicas de análisis y planificación de programas de enseñanza aprendizaje; c) la administración operativa y la coordinación de los sistemas comunicaciones y programas de enseñanza aprendizaje; d) la evaluación de los resultados de los esfuerzos educacionales (sistemas, escuelas, programas, alumnos, personas docente, etc.); e) la integración y utilización efectiva e los nuevos desarrollos en las áreas de comunicaciones masivas. (p. 161).

dos, según el área mayor de la especialidad-, Licenciatura en Áreas Técnicas, Licenciatura en Ciencias Sociales, y Licenciatura en Técnicas Audiovisuales y Psicopedagogía.

4 Ver decretos 1710 de 1963, y 1955 de 1963.
Al respecto, es indispensable señalar que, para Chadwick, hay dos maneras de asumir la te: la primera, en su manifestación en forma de máquina y otros dispositivos que se pueden usar en el proceso educativo, y la segunda, como aplicación del conocimiento organizado o científico a la resolución de problemas prácticos en la educación (Chadwick, 1983). En este sentido, las áreas que van a predominar en la te son: la comunicación, la psicología y el enfoque de sistemas; que deberían -en la práctica- mantener un equilibrio. Este apunte resulta relevante en el sentido de que la apropiación que se evidencia en la FCE ha sufrido variaciones a lo largo del tiempo, tanto en sus programas de estudios, como en la forma en que es asumida por quienes cursan sus estudios.

Un ejemplo de ello se encuentra en el pénsum de Licenciatura Técnicas Audiovisuales y Psicopedagogía, con el que funcionó desde 1971 hasta 1978, en el que se incluian asignaturas como Historia de los Medios de Comunicación; Ayudas Educativas; Grabación de Sonido; Manejo de Equipos; las cuales estaban ligadas a la idea del uso de dispositivos en el proceso de enseñanza aprendizaje. Cabe mencionar que las asignaturas de componente pedagógico como: Sociología de la Educación; Psicología General; Historia y Filosofía de la Educación; Didáctica General, y Psicología del Desarrollo (UTP, 1970) ${ }^{5}$, y para sus tres semestres de estudios profesionales ${ }^{6}$ contaba además con Psicología Educativa, Sicología Dinámica y Currículum. Esta programación académica estaba ligada a lo que Chadwick ha definido como el modelo clásico y la enseñanza audiovisual, en el que el propósito fundamental fue ofrecer mejores técnicas para que el profesor pudiera utilizarlas en el aula, alli las ayudas por medio de aparatos era un imperativo e iban desde diapositivas hasta películas y cintas grabadas. Por medio de estos cambios, se van tramitando transformaciones importantes para el hecho educativo, pues no solo cambia el rol del docente ya que involucra a sus estudiantes

5 Estas asignaturas estaban incluidas en el pénsum común a todas las licenciaturas de la Facultad, que era desarrollado en los dos primeros semestres académicos de estos programas, lo que se conoció en su momento como Estudios Básicos.

6 Para la fecha se hablaba de programas de expertos que, según la reglamentación del Decreto 1964 de 1969 del Ministerio de Educación, correspondian a programas de profesionalización de duración media, de setenta créditos, y en los cuales a los dos primeros semestres -los estudios básicos- seguian tres semestres de estudios profesionales enfocados en la formación disciplinar. 
mediante la participación en el aula, sino que los contenidos son variados con énfasis en el procesamiento y de la solución de problemas (Chadwick,1997, p. 55).

En consonancia con lo anterior, llama la atención que muchos de los docentes que lideraron las primeras propuestas y sus posteriores transformaciones se formaron bajo el nuevo modelo en la Universidad de Florida, Tallahassee, como lo señala el Master of Science Fernando Maldonado Delgado 7 . El profesor Maldonado que se formó en la Licenciatura en Técnicas Audiovisuales y Psicopedagogía también recuerda la visita de un italiano -hermano Parodi Merlino- quien aportó a la transformación del área del programa académico en mención, hacia el programa de Licenciatura en Español y Comunicación Audiovisual, que fue aprobado por dos años -por la Resolución No. 1774 del 14 de marzo de 1978, del Ministerio de Educación Nacional (UTP, s.f.)-.

El papel de este profesor italiano fue significativo en la construcción del programa de Licenciatura en Español y Comunicación Audiovisual de la uTP. Este docente habia Ilegado desde Chile en 1972, bajo la modalidad de intercambio a la Universidad del Quindío Fundada en 1961, según lo que relató el director del Centro Audiovisual de dicha institución de educación superior -licenciado Hernán Prieto Torres- para el diario regional Crónica del Quindío en 2013:

Hermano Parodi Merlino -por su nombre, también confundido como religioso- atendió el llamado del Programa de las Naciones Unidas para el Desarrollo, PNUD, y llegó a la Universidad, con su parafernalia tecnológica. Se inició entonces la microfilmación de las clases para el perfeccionamiento de la didáctica de los docentes de la primera carrera de ese centro de educación superior: la licenciatura en pedagogía y administración educativa.

Lo anterior guarda estrecha relación con la consolidación de la línea de TE en la FCE. Porque fue precisamente a mediados de la década de los setenta cuando se daba apertura al discurso de la TE que provenía de las políticas internacionales. Al respecto, el profesor Maldonado comenta:

7 Egresado de la Licenciatura en Psicopedagogia y Técnicas Audiovisuales en 1975 posteriormente se desempeñó como docente de la Licenciatura en Comunicación y Español Audiovisual.
Por otro lado, llegó a Colombia de una misión europea el Hermano Parodi, italiano, y él tenía la concepción de la comunicación educativa pero más como tecnología. Y el primer programa que nos ayudó a pensar o a diseñar fue en tecnología educativa. Se hizo un convenio con la Universidad, pero luego fue derivando hacia el área de la comunicación, más pensando en esto de formación de imaginarios colectivos y de cultura. (Comunicación personal, septiembre de 2015).

En esta transición de la Licenciatura en Técnicas Audiovisuales y Psicopedagogía hacia la Licenciatura en Español y Comunicación Audiovisual, es posible evidenciar la primera ruptura que tiene que ver con el naciente Decreto 1419 de 1979 que promulgaba la "Renovación Curricular". Su fundamento estuvo centrado en alcanzar la eficacia del proceso educativo, con lo que se empezaría a plantear la importancia de la planificación en el currículo. En este sentido, se profundiza en la FCE el componente de la comunicación educativa en lo que tiene que ver con la elaboración de materiales didácticos para realizar actividades. Con la puesta en marcha de este nuevo programa se puso de manifiesto que la pedagogía estaba siendo relegada por el componente disciplinar de la Licenciatura: la comunicación educativa, forma en que se apropió en la FCE la TE.

Para ilustrar mejor, se mencionan a continuación las asignaturas que componían el pénsum de la nueva Licenciatura en Español y Comunicación Audiovisual (UTP, 1994)8: Historia de la Pedagogía; Epistemología, y Escuelas Sicológicas; Procesos Sicológicos; Aprendizaje: problemas, dificultades y tratamientos; Didáctica Especial; Evaluación; Taller de Docencia. En el área disciplinar se encontraban: impresos; radio; cine; producción audiovisual; fotografía; industrias culturales, proceso de democratización de la participación en medios; lengua y uso; exploración visual y fotoperiodismo. Dentro del programa de Licenciatura en Español y Comunicación Audiovisual, aprobado en 1978, el objetivo de formación de docentes en esta disciplina fue:

8 Como ya se trataba de un programa profesional, los estudios en la Licenciatura en Español y Comunicación Audiovisual eran independientes de las demás licenciaturas que se ofertaban en la Facultad. Adicional a esto, el programa tenía ya una duración de diez semestres académicos, en los cuales los componentes pedagógicos y disciplinar se desarrollaban simultáneamente. 


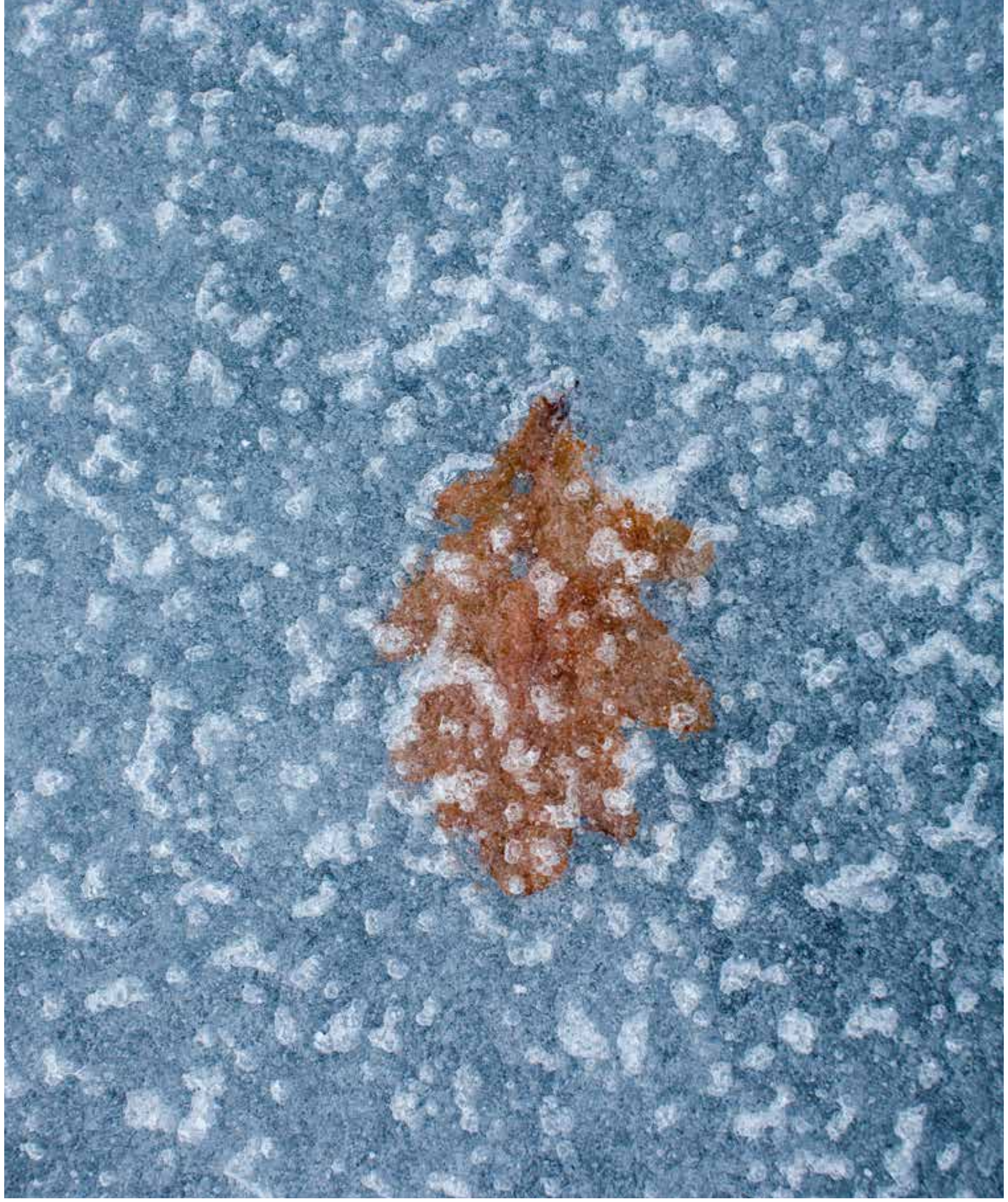

Autora: Ileana Álvarez Reyes

Título: Sprut av Liv

Lugar: Østmarka, Oslo, Noruega 
Formar un profesor de Español que desde el saber pedagógico integre los diversos campos del conocimiento comprometidos en el estudio de la naturaleza, estructura, funcionamiento, usos y relaciones de los distintos lenguajes que posibilitan los procesos cognitivos, estéticos e interactivos, desde varios enfoques científicos. (UTP, 1994).

En tal sentido, para este programa se contó con un equipo interdisciplinario de docentes, especializados unos en la lingüística -como es el caso de Rafael Areiza y Nelson Goyes Ortega ${ }^{9}$ - otros en la literatura - un caso puntual es el profesor César Valencia Solanilla, quien llegaria a la Facultad en el año de 1994-, y en el área de comunicación audiovisual, estuvieron profesores como Fernando Maldonado, Amanda Castiblanco, Ligia Méndez y Virginia Aristizábal, quienes evidenciarian fuertes vacíos teóricos y prácticos en este área, por lo cual empezaron a estudiar los planteamientos de autores como Armando Silva Téllez, Jesús-Martín Barbero, Omar Rincón, Néstor García Canclini, lo que dio origen a una serie de reflexiones sobre un campo de estudio interdisciplinar que, para ellos, tendría que consolidarse en la Licenciatura -la comunicación educativa-; alli se pondría el acento de la TE provocando a su vez una brecha entre el equilibrio de las disciplinas que la componen (comunicación, psicología y teoría de sistemas) en el interior de la Facultad. Cabe mencionar que dentro de las líneas de trabajo de esta licenciatura estaba la de "ciudad", ligada a la comprensión, por parte del estudiante, de la tríada sociedad, cultura y comunicación, en función de formarse como ciudadanos de mundo.

Sobre este particular, la profesora jubilada Amanda Castiblanco Cardona manifestó que, en la Licenciatura en Español y Comunicación Audiovisual se estudiaban las

[...] teorias de la comunicación [de manera] muy incipiente, algunas asignaturas de medios, y unos proyectos que se hacian integrando las demás áreas [literatura y lingüistica] con un proyecto audiovisual, donde confluia interdisciplinariamente, es decir, lo que habia en lingüistica,

9 El profesor Luis Nelson Goyes Ortega también fue decano de la Facultad para el periodo de 1987, sucediendo así en este cargo a la Ruth Rodríguez de Zuluaga, profesora del Departamento de Matemáticas, quien sería una pieza clave en la consecución de la aprobación para el primer programa de posgrado de la Facultad: la Maestria en Comunicación Educativa, para 1986. literatura y lo que pasaba por las asignaturas de comunicación. (Amanda Castiblanco Cardona, comunicación personal, 2016).

De esta forma, en el seno de este programa académico se presentó una nueva modificación, en términos, tanto en la continuidad de los programas académicos como en la concepción de la TE; la cual se entendía tanto desde el diseño instruccional del currículo, como desde los aparatos tecnológicos que podrían dinamizar el proceso educativo, pero en esta fractura le empezó a dar mayor relevancia a la concepción como aparato tecnológico. Lo anterior se decanta con la creación de la Maestría en Comunicación Educativa en 1986, como una respuesta a las necesidades de especialización que planteaba la nueva reglamentación educativa oficial en el país. Esta fractura tiene su origen en la llamada Renovación Curricular que se promovió luego de la publicación del Decreto 1002 de 1984 que planteaba:

Artículo $6^{\circ}$. En la Educación Básica Secundaria, además de las áreas anteriores, se incluyen como áreas comunes la de educación en tecnología y la correspondiente a un idioma extranjero. Parágrafo. Se entiende por educación en tecnología la que tiene por objeto la aplicación racional de los conocimientos y la adquisición y ejercicio de habilidades y destrezas que contribuyan a una formación integral, faciliten la articulación entre educación y trabajo, y permitan al alumno utilizar de manera efectiva los bienes y servicios que le ofrece el medio.

Artículo $7^{\circ}$. El área de educación en tecnología, que cada centro educativo establecerá en Básica Secundaria, debe seguir una secuencia organizada y mantener la debida continuidad a través de todos los grados. Esta área estará preferentemente orientada hacia la o las modalidades elegidas por el Centro Educativo.

En función de esta legislación cobra relevancia no solo la existencia de este campo educativo en la FCE, sino también en cómo sería abordada la formación de los futuros docentes.

\section{La comunicación educativa: adecuación de la TE}

En el periodo comprendido entre 1998-2002, luego de la publicación del Decreto 272 de 1998, la FCE dio inicio a un proceso de revisión exhaustiva de los programas académicos que ofertaba, por lo que se decidió 
no presentar la Licenciatura en Español y Comunicación Audiovisual al proceso de Certificación de Calidad, sino empezar el proceso de reformulación, es decir, dividirla en dos nuevas carreras. Comentan los profesores Gonzaga Castro Arboleda (comunicación personal, 2016) y Rigoberto Gil Montoya (comunicación personal, 2016), dos factores que imperaron en esta decisión administrativa.

En primer lugar, el hecho de tener un alto número de egresados de la Licenciatura en Español y Comunicación Audiovisual empezaba a ser problemático, dado que muchos licenciados estaban afrontando dificultades para posicionarse satisfactoriamente en el campo laboral de acuerdo a su perfil profesional y al exceso de producción de licenciados en esta área. En segundo lugar, la consolidación de la Maestría en Comunicación Educativa que, como ya se afirmó, surgió porque algunos docentes evidenciaron ciertas falencias en la reflexión y formación sobre la comunicación audiovisual, permitió dar cuenta de la necesidad de separar los componentes disciplinares de la Licenciatura en Español y Comunicación Audiovisual, los cuales hasta el momento serían la literatura, la tecnología y la comunicación educativa, como se verá en el siguiente fragmento:

En sesión del Consejo Superior del 11 de marzo del 2002, se aprueba por unanimidad el proyecto de Licenciatura en Comunicación e Informática Educativas, Nuevas Tecnologias de la educación y la información para educación en nuevos entornos culturales. Esta propuesta es un desarrollo del antiguo programa de Licenciatura en Español y comunicación audiovisual, el cual recoge los acervos teóricos institucionales acumulados durante décadas. Este se vincula al área de comunicación educativa y en la filosofia de ciencia y tecnología "la potencialización de los medios de comunicación audiovisual como instrumentos de desarrollo". El proyecto posee como contenido: la definición, los objetivos, la fundamentación legal, jornada, perfil profesional, las áreas de formación, los docentes de tiempo completo, (son seis docentes, en su gran mayoría con maestria), el pensum académico y la homologación con la Licenciatura en Español y Comunicación Audiovisual. (...) Dentro de los antecedentes históricos: La universidad posee unos acervos teóricos que permiten el desarrollo del presente proyecto, como "el antiguo programa de Licenciatura en Psicopedagogía y Técnicas Audiovisuales; La Escuela de Español y Comunicación Audiovisual, y su respectiva anterior licenciatura que lleva su nombre; La Maestría en Comunicación Educativa, La participación en el proyecto de Televisión Educativa Iberoamericana y otros proyectos, El Centro de Recursos Educativos e Informáticos (CREI), El Centro de documentación Virtual de la FCE y la Red inter e intra Institucional de correo electrónico, el Centro de Televisión y Emisora Universitaria y la Ingeniaría en Sistemas de la Información. (UTP, 2002).

Por lo anterior, finalmente este programa cerró sus inscripciones de manera definitiva, para dar paso a la creación de la Licenciatura en Español y Literatura -estructurada, principalmente, por el profesor César Valencia Solanilla- en 2001, haciendo énfasis en los componentes lingüísticos y literarios, y de la Licenciatura en Comunicación e Informática Educativas -construida por el profesor Fernando Maldonado Delgado y Ricaurte Murillo- en 2002, centrada en la TE conjugando dos elementos: la comunicación y la informática educativas. Cabe señalar que quienes formularon el proyecto educativo de este nuevo programa apuntaban a la formación de un profesional de la educación especializado en la construcción de proyectos educativos mediados por la tecnología, en procesos de educación no formal e informal ${ }^{10}$. Sin embargo, la Licenciatura que finalmente se aprobó, puso el énfasis en la formación de un profesional que atendiera por medio de las Nuevas Tecnologías de la Comunicación y la Información (NTIC), las necesidades de la educación media del sistema educativo formal. Así, se ha desarrollado este programa académico

10 Habría que mencionar que, para el año de 1992, el profesor Maldonado presentó un proyecto para la creación del Pregrado -Licenciatura- en Comunicación Educativa, retomando como el principal antecedente su experiencia -con el licenciado en Español y Comunicación Audiovisual, Alberto Henao Campusano- en el Ingenio Riopaila S.A., con un proyecto educativo denominado Plan para recuperarycompartir información tecnológica de sus divisiones industrial y de maquinaria. En tal sentido, esta propuesta de programa académico estuvo enfocada a atender las demandas de la sociedad de la época, traducidas en la apertura económica y los cambios científico-tecnológicos de finales del siglo xx, que requerían de profesionales de la educación competentes en la formulación y ejecución de proyectos de capacitación de los trabajadores de la industria regional. Evidentemente, el enfoque con el cual el profesor Maldonado abordaba la comunicación educativa $-y$, por consiguiente, la construcción del perfil profesional del egresado de una Licenciatura en este árease situaba en el terreno de la educación no formal e informal. Este programa fue aprobado en el Acta 20 del 27 de octubre de 1993 por el Consejo Académico y, en consecuencia, se sugirió al Consejo Superior que se aprobara y tramitara la respectiva licencia de funcionamiento ante el ICFES. Finalmente, y según mencionó Fernando Maldonado, el Consejo Superior decidió rechazar este proyecto, dado que los recursos económicos que se destinaban para la Facultad de Ciencias de la Educación serian ejecutados para los programas académicos que ya estaban en funcionamiento. No obstante, es importante entender esta Licenciatura como un referente especial para la propuesta que presentó el mismo profesor durante la rectoria del ingeniero Luis Enrique Arango Jiménez, y que sería aprobada en 2002 con el énfasis en el nivel medio de la educación formal. 
desde sus inicios hasta la actualidad, dentro de su programa académico continúa en la conservación de asignaturas como cine, televisión, radio, fotografía, video y diseño gráfico, todas ellas abordadas con un enfoque educativo en función del diseño de nuevos ambientes de aprendizaje.

\section{Consideraciones finales}

Como se vio, la te ha tenido algunas variaciones y en particular en la Facultad de Ciencias de la Educación de la UTP, el desarrollo de este campo puso el énfasis en la comunicación educativa en el programa de Licenciatura en Comunicación e Informática Educativa, en el que hay un predominio del componente disciplinar reflejado en el uso de los medios de comunicación (radio cine, televisión, fotografía, diseño gráfico, video, cursos de maestro virtual, nuevos formatos audiovisuales e informática). Estas asignaturas, en términos de créditos, están por encima del componente pedagógico (ambientes educativos, teorías cognitivas, evaluación del aprendizaje, etc.).

La Licenciatura en Comunicación e Informática Educativa cuenta en la actualidad con dos procesos de acreditación, en los cuales ha obtenido la calificación de Alta Calidad. Sin embargo, después de que el Ministerio de Educación Nacional reformara el mecanismo para la obtención del registro calificado de los programas de licenciatura y su posterior acreditación de calidad -con la publicación de la Resolución 02041 de 2015 posterior Resolución 18583 de septiembre de 2017, legislación en la cual tal denominación no cumplía con lo requerido por el MEN-, su comité curricular, en cabeza del doctor Arbey Atehortúa. Atehortúa, inició las discusiones sobre el nuevo título que iba a ofertarse como las estrategias que utilizarian para llevar a feliz término a quienes a la fecha estaban inscritos en la Licenciatura en Comunicación e Informática Educativa.

Conviene señalar que esta situación no fue exclusiva de esta licenciatura, sino que fue una tensión que se presentó en todas las universidades del país que ofertan estos programas, pues la intención del Ministerio fue generar reglamentar y organizar las denominaciones para los programas de licenciatura que correspondieran con las áreas de educación básica y media consignadas en la Ley General de Educación, como también dar claridad sobre los componentes específicos y las competencias de los educadores en formación.

Para abordar esta nueva reforma, bajo la asesoría de la Vicerrectoría Académica de la Universidad y a su vez del Ministerio de Educación Nacional, el comité curricular de la Licenciatura en Comunicación e Informática Educativa formuló un nuevo programa que cumpliera con las demandas de la naciente Resolución. En esta perspectiva se ven reflejadas las continuidades o el imperativo de una escuela por mantener una línea disciplinar en esta Facultad, la de la TE. El nuevo programa (que ya fue aprobado por el csu de la institución) se denomina Licenciatura en Tecnología con énfasis en Comunicación e Informática Educativa, que en el plan de estudios conserva en un alto porcentaje el pénsum anterior, y que sus cambios son la inclusión y fortalecimiento del área de prácticas docentes e investigación.

Sin duda, la pertinencia de este programa en un mundo globalizado que ha puesto a la mayoría de la población en la lógica del uso y el consumo de tecnologías. Se podria decir incluso que el Proyecto Multinacional para la te tuvo un efecto positivo en términos de su implantación en Colombia, pues la UTP no fue la única en asumir esta línea disciplinar, sino que fue un proceso en el que participó con mucha más fuerza la Universidad Pedagógica Nacional y la experiencia del uso de ciertos dispositivos como la radio con Radio Sutatenza, o la televisión educativa, se lograron mejorar la calidad y el acceso de las sesiones de encuentro entre docentes y estudiantes en todos los niveles.

Al respecto, el licenciado en Español y Literatura Farid García Ramírez considera que dentro de la comprensión de la TE en la FCE-UTP, es importante seguirle el rastro a las innovaciones tecnológicas y curriculares. En este sentido, se tendría que dirigir la mirada hacia el impacto que estas discusiones han tenido en los estudiantes. Esto podría otorgarle nuevos matices al análisis, en la medida en que pudieron ser muchos los estudiantes que participaron en los debates frente a la comunicación y las nuevas tecnologías, pero también había quienes únicamente se 
interesaban por aprobar las asignaturas del plan de estudios, lo cual ya se constituía en el propósito de las mayorías. En este sentido, es evidente una racionalidad instrumental, si se tiene en cuenta que pocos estudiantes tenían la convicción de lo que significaba formarse profesionalmente -y en el campo de la tecnología educativa-. Por lo demás, esa profesionalización en palabras del egresado en mención: "[...] fue un coletazo de docentes del decreto antiguo, que necesitaban obtener su escalafón siete".

Hay que mencionar además que, entre los aspectos relevantes en la construcción del campo de aplicación de la te se encuentra el análisis de la creación de Telecafé y de la Maestría en Comunicación Educativa, los que se entenderían como procesos institucionales. Sin embargo, habria que dar cabida también a procesos que no figuran en las actas y documentos oficiales, y que tienen que ver con el crecimiento cultural que empezaba a gestarse en la región, con el surgimiento de cineclubes y otros escenarios en los que se acudía al video como herramienta, o procesos educativos sociales y comunitarios.

Sin embargo, y siguiendo la línea argumental de Alberto Martínez Boom, la reforma en la formación de docentes debe estar basada en su experiencia, pues,

[...] cuando la formación apuesta por construir la experiencia del maestro, cualquiera que sea el umbral en que aquella se desarrolle, se convierte en una disposición ética y cultural que ayuda al maestro a movilizar su pensamiento, a pensarse de otras maneras y a asumir su quehacer como sujeto potente. (Martínez Boom, 2016b, p. 120).

En la FCE, siendo la TE una línea temática que lleva 46 años de existencia, habrá que hacer una revisión sobre su vigencia y pertinencia, el impacto de esta formación de docentes en comunicación e informática educativa, que efectivamente responda al diseño y desarrollo de ambientes de aprendizaje que incorporen las ayudas educativas en función del desarrollo del aprendizaje en un amplio rango de cobertura, sea en el aula o en la generación de contenidos audiovisuales. Todo ello plantea también la importancia de reflexionar sobre el problema que debe resolver un licenciado en Tecnología en la sociedad actual, para así otorgarle un sentido a este tipo de formación, que demande la solución a otras crisis o problemas que hoy tiene la educación pero también para que el desarrollo del proceso educativo no dependa en gran medida de las visiones que construyen los organismos internacionales, como otrora lo planteara el Movimiento Pedagógico ${ }^{11}$.

\section{Referencias}

Acevedo, T., Gutiérrez, N. y Rodríguez, D. (2009). Jorge Roa Martínez: memorias de una visión cosmopolita. Pereira: Editorial Universidad Tecnológica de Pereira

Atcon, R. (1963). La universidad latinoamericana: clave para un enfoque conjunto del desarrollo coordinado, social y educativo en América Latina. Bogotá: ECOE Ediciones.

Chadwick, C. (1983). La tecnología educativa en América Latina en la década de los setenta. Educar, 3, 129-141.

Chadwick, C. (1997). Tecnología educacional para el docente. Barcelona: Paidós.

Chadwick, C. (2003). Análisis teórico de la tecnología educativa. En A. Martínez Boom, C.E. Noguera y J.O. Castro, Currículo y modernización: Cuatro décadas de educación en Colombia. Bogotá: Cooperativa Editorial del Magisterio.

Coombs, P. (1971). La crisis mundial de la educación. Barcelona: Ediciones Peninsula.

Departamento Nacional de Planeación de la República de CoIombia (1975). Para cerrar la brecha: Plan de Desarrollo Social, Económico y Regional 1975-1978. Bogotá.

La Crónica del Quindio (5 de enero de 2013). Centro audiovisual, 40 años apoyando la docencia, la investigación y la extensión. [En línea]. Recuperado de http://www. cronicadelquindio.com/noticia-completa-titulo-centro_audivisual__40_anos_apoyando_la_docencia__ la_investigacion_y_la_extension-seccion-general-nota-56461.htm

Martínez Boom, A. (2016a). Maestro, función docente y escolarización en Colombia. Propuesta Educativa, 1(45), 34-49.

Martínez Boom, A. (2016b). La formación en las universidades pedagógicas. Un punto de encuentro. Nodos y Nudos, 4(40), 117-122.

Martinez Boom, A., Zuluaga, O., Echeverry, A., Restrepo, S. y Quiceno, H. (1988) Educación y Pedagogía; Una diferencia necesaria. Revista Educación y Cultura, 14,4-9.

11 Alberto Martínez Boom plantea en la revista Educación y Cultura, de FECODE, de julio de 1988 que el Movimiento Pedagógico, aunque manifestó un rechazo a las propuestas de organismos internacionales, durante este periodo, no fue como tal a la tecnología educativa per se, sino a la renovación curricular y al papel que ellos como docentes jugarian posteriormente en el sistema educativo, el de simples ejecutores. 
Martínez Botero, S. y Correa, J.J. (2016). La Facultad de Ciencias de la Educación de la Universidad Tecnológica de Pereira (1967-2017): sus inicios, permanencias $y$ contrastes. Ponencia en el xl Congreso Internacional de Historia de la Educación Latinoamericana SHELA, 17 al 19 de octubre. Universidad San Carlos de Guatemala.

Martínez Boom, A., Noguera, C.E. y Castro, J.0. (2003). Currículo y modernización: cuatro décadas de educación en Colombia. Bogotá: Cooperativa Editorial del Magisterio.

Patiño, R.J. (1966). Hacia la universidad del desarrollo: bases de una politica de reforma universitaria. Bogotá: Universidad Nacional de Colombia.

Pérez, D.A. (coord.) (s.f.). Transferencia de Tecnología Educativa en Colombia. Bogotá: Centro Editorial Don Bosco.

Presidencia de la República. Decreto 088 de 1976, por el cual se reestructura el sistema educativo y se reorganiza el Ministerio de Educación Nacional. Bogotá.

Presidencia de la República. Decreto 1002 de 1984, por el cual se establece el Plan de Estudios Para la Educación
Preescolar, Básica (Primaria y Secundaria) y Media Vocacional de la Educación Formal Colombiana. Bogotá.

Presidencia de la República. Decreto 272 de 1998 por el cual se establecen los requisitos de creación y funcionamiento de los programas académicos de pregrado y posgrado en Educación ofrecidos por las universidades y por las instituciones universitarias, se establece la nomenclatura de los títulos y se dictan otras disposiciones. Bogotá.

Universidad Tecnológica de Pereira (UTP) (1965). Acta 90. Pereira: Consejo Directivo de la UTP.

Universidad Tecnológica de Pereira (UTP) (1970). Acta 15. Pereira: Consejo Directivo de la UTP.

Universidad Tecnológica de Pereira (UTP) (1994). Anexo al Acta 09. Propuesta de reforma curricular [de los programas de la Facultad de Ciencias de la Educación]. Pereira: Consejo Académico de la UTP.

Universidad Tecnológica de Pereira (UTP) (2002). Acta 01. Pereira: Consejo Superior Universitario de la UTP.

Universidad Tecnológica de Pereira (UtP) (s.f.). Anexo al Acta 008. Pereira: Consejo Superior Universitario de la UTP. 


\section{DIÁLOGO DEL CONOCIMIENTO}

El reto de enseñar en, para y desde la tecnología implica varios presupuestos que van desde la comprensión y evolución de los sistemas mecánicos, entendimiento de aspectos relacionados con

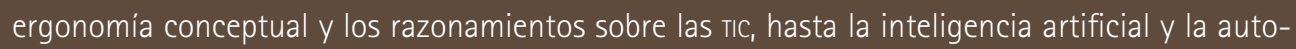
matización, como grandes marcos de acción en el campo de la enseñanza y aprendizaje de lo tecnológico. Sin embargo, hay tradiciones tanto académicas como del sector productivo que pretenden simplificar la tecnología a los artefactos, vincularla exclusivamente a las prácticas del capitalismo o llevarla al mundo de un pragmatismo irreflexivo, sirviendo al sector bélico o de intereses perversos. La tecnología entonces antes menospreciada, por su naturaleza práctica, frente a la filosofía u otras ciencias, hoy propone reflexiones; formula problemas éticos, estéticos, y por supuesto, proyecta escenarios epistémicos que van más allá de lo técnico.

Hoy los más destacados artistas están diseñando escenarios y personajes para videojuegos; los economistas están repensando el problema de la circulación de capital a propósito de las criptomonedas o el e-commerce; los sectores como transporte, medios de comunicación, entre otros, han variado y reformulado sus naturalezas, a partir del uso y las demandas de la tecnología. Por su parte, la educación en este campo ha estado en una constante transformación, por ello, no hay consenso respecto de los contenidos para la enseñanza de tecnología. Toda labor o propuesta educativa proyecta nuevas tendencias y descubre otras posibilidades, por ello, las investigaciones propuestas desde la academia reformulan miradas y emprenden nuevos caminos que, sin duda, propenden por mejores personas en una mejor sociedad.

Una academia que cumple 50 años, como la Facultad de Ciencias de la Educación de una universidad pública como la Tecnológica de Pereira, da cuenta de la tradición, el esfuerzo, la disciplina y la dedicación de colectivos intelectuales y reflexiones particulares sobre este campo. Congratulaciones, por esta importante labor que pretende pensar y repensar lo que hacemos y lo que somos, y los aportes y nuevas posibilidades que surgen para conservar el legado material e inmaterial, para el fomento de pensamiento crítico y para fortalecimiento de la democracia.

Carlos Hernán López Ruiz Coordinador del Centro de Innovación y Desarrollo Educativo y Tecnológico (CIDET) Docente de la Facultad de Ciencia y Tecnología Universidad Pedagógica Nacional 